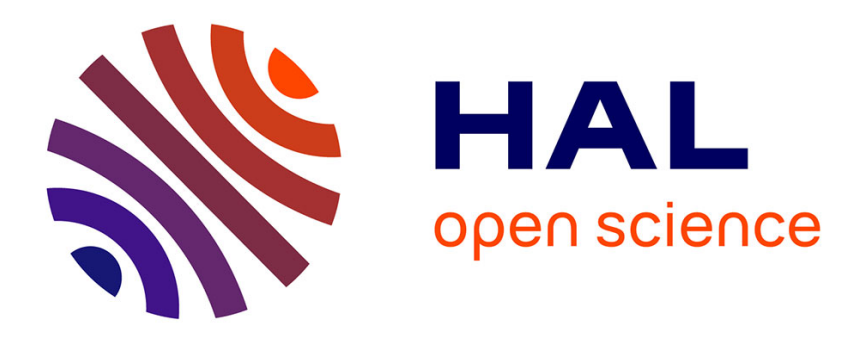

\title{
Positron Methods for the Study of Defects in Bulk Materials
}

\author{
M. Eldrup
}

\section{To cite this version:}

M. Eldrup. Positron Methods for the Study of Defects in Bulk Materials. Journal de Physique IV Proceedings, 1995, 05 (C1), pp.C1-93-C1-109. 10.1051/jp4:1995111 . jpa-00253547

\section{HAL Id: jpa-00253547 https://hal.science/jpa-00253547}

Submitted on 1 Jan 1995

HAL is a multi-disciplinary open access archive for the deposit and dissemination of scientific research documents, whether they are published or not. The documents may come from teaching and research institutions in France or abroad, or from public or private research centers.
L'archive ouverte pluridisciplinaire HAL, est destinée au dépôt et à la diffusion de documents scientifiques de niveau recherche, publiés ou non, émanant des établissements d'enseignement et de recherche français ou étrangers, des laboratoires publics ou privés. 


\title{
Positron Methods for the Study of Defects in Bulk Materials
}

\author{
M. Eldrup \\ Materials Department, Ris $\phi$ National Laboratory, DK-4000 Roskilde, Denmark
}

\begin{abstract}
The basic principles of positron annihilation physics are briefly discussed and the three most important experimental techniques used for bulk studies are described (i.e. positron lifetime, angular correlation, Doppler broadening). Several examples of the use of the positron methods are discussed for metals, ceramics and molecular materials, which illustrate the sensitivity of the positron annihilation techniques to vacancy type defects. For example it is shown how information can be obtained about vacancy formation energies, vacancy migration and clustering, vacancy-impurity interactions, densities of rare gasses in bubbles in metals, and about free volume in molecular materials.
\end{abstract}

\section{INTRODUCTION}

The positron is the antiparticle to the electron. This means that the positron has the same mass and spin as the electron, but has the opposite charge, viz. one positive elementary charge. Furthermore, if a positron is surrounded by one or more electrons the positron may annihilate with one of the electrons, i.e. both particles disappear and their masses are transformed into energy which is emitted as $\gamma$-quanta, normally two or three. The properties of these $\gamma$-quanta, such as their energies, emission directions, and time of emission which can all be measured, provide useful information about the material in which the positrons annihilate. Very briefly, this is the principle of the Positron Annihilation Spectroscopy.

Positron Annihilation research is a very wide field as can be judged from the proceedings of the most recent international conferences [1-5] and other recent publications [6-10].

In the present paper we shall concentrate on a discussion of the possibilities to study defects in solids, by giving examples for metals, ceramics and molecular materials. First, however, let us discuss some of the basic physical principles of positron annihilation and then describe the most important experimental techniques used in positron annihilation studies of defects in bulk materials. 


\section{POSITRONS IN SOLIDS}

In conventional positron annihilation experiments the positrons are injected into a solid with a mean energy of the order of $200 \mathrm{keV}$. They slow down to thermal energies in about $10^{-12}-10^{-11} \mathrm{sec}$ (1-10 psec) by ionisation and excitation of the solid. During this time they penetrate a distance of 10-1000 $\mu \mathrm{m}$ depending on the density of the solid (the penetration depth is roughly inversely proportional to the density). Hence, the positrons probe bulk material in such experiments. In recent years a rapid development of a new technique has taken place, viz. of low-energy-positron beams. This technique will be discussed by others at this workshop [11].

\subsection{Annihilation of Positrons}

A positron that has been injected into a solid and has slowed down, in most cases annihilates with the emission of $2 \gamma$-quanta, since for free positrons the cross-section for emission of $3 \gamma$ is only $1 / 379$ of the $2 \gamma$ cross-section. The two annihilation quanta have a total energy of $E=2 \mathrm{~m}_{0} \mathrm{c}^{2}$ $=2 \times 511 \mathrm{keV}\left(\mathrm{m}_{\mathrm{o}}\right.$ is the electron or positron rest mass, $\mathrm{c}$ the velocity of light) and are emitted in almost opposite directions as sketched in Fig. 1. The angle $\Theta$ is usually very small, typically $<$ 20 milliradian ( $\mathrm{mrad})\left(20 \mathrm{mrad} \approx 1^{\circ}\right)$. This is so, because the total momentum of the annihilating positron-electron pair has to be conserved in the momentum of the two quanta. If this momentum deviates from zero, the directions of emission of the two quanta will deviate from co-linearity (Fig.1). In a good approximation the angle between them is given by:

$$
\Theta=\mathrm{p}_{\mathrm{T}} / \mathrm{m}_{\mathrm{o}} \mathrm{c}
$$

where $\mathrm{p}_{\mathrm{T}}$ is the total momentum of the $\gamma$-quanta.

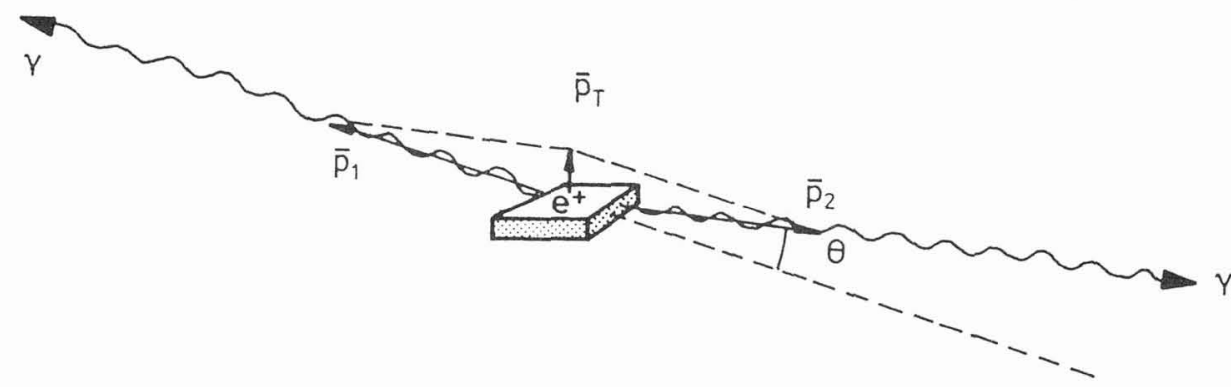

Fig.1. Emission of two annihilation $\gamma$ quanta in almost opposite directions. The vectors represent the momenta of the two quanta $\left(\bar{p}_{1}\right.$ and $\left.\bar{p}_{2}\right)$ and the total momentum of the pair $\left(\bar{p}_{\tau}\right)$. The angle $\Theta$ is usually very small, typically $\Theta<1^{\circ}$.

In many cases, in particular in metals, the positron momentum is low compared to the electron momentum. Hence, a measured momentum distribution is essentially that for the electrons. Such measurements are being used to determine the electronic structure of metals and alloys [12-14]. The rate with which positrons annihilate with the surrounding electrons is given by [15]:

$$
\lambda=\pi r_{0}^{2} c \int \rho^{-}(\bar{r}) \rho^{+}(r) d r
$$

where $r_{0}$ is the classical electron radius $\left(=\mathrm{e}^{2} / \mathrm{m}_{0} c^{2}\right)$ and $\rho^{+}$and $\rho^{-}$are the positron and total electron density at $\bar{r}$, respectively. From Eq. (2) we see that the positron mean lifetime (normally just referred to as the lifetime) $\tau=\lambda^{-1}$ is a measure of the electron density sampled by the positron, in the sense that a lower electron density results in a longer positron lifetime. 


\subsection{Positrons in Metals}

Generally speaking, the behaviour of positrons in metals is simpler and better understood that the behaviour in other solids. Let us therefore first consider the former. In a perfect metal lattice the positron will, after slowing down, diffuse around, normally in a delocalized, Bloch-function, state. Positron lifetimes in metals are 100-400 psec, depending upon the metal [16]. During its lifetime the positron typically diffuses about $200 \mathrm{~nm}$. Figure $2 \mathrm{~A}$ illustrates the positron density in a perfect lattice.

If the metal contains defects such as vacancies, vacancy cluster and dislocations, i.e. regions of less than average density, positrons may become trapped, i.e. localized at these defects. This is because the positron is repelled by the positively charged ion cores. Hence, structural defects which represent missing (or a reduced density of) ions will provide attractive potentials for positrons (see e.g. [17] for a more detailed description of the trapping potential). Figure 2B illustrates the density of a positron trapped at a vacancy. Trapped in such a defect the positron will experience a lower electron density than in the bulk material and its lifetime is therefore increased (Eq. (2)). Furthermore, the average conduction electron momentum at the defect is lower than in the bulk and the positron overlaps less with the high-momentum core electrons. Both these effects lead to a narrower total-momentum distribution for the annihilation quanta. These changes in annihilation characteristics for defect-trapped positrons (which can be measured; see next section) are the basis for the now well established use of PAS to metal defect studies. It should be noted in particular that positrons are not trapped by interstitials or small clusters of these. Thus, the positron is specifically sensitive to vacancy type defects (low density regions).
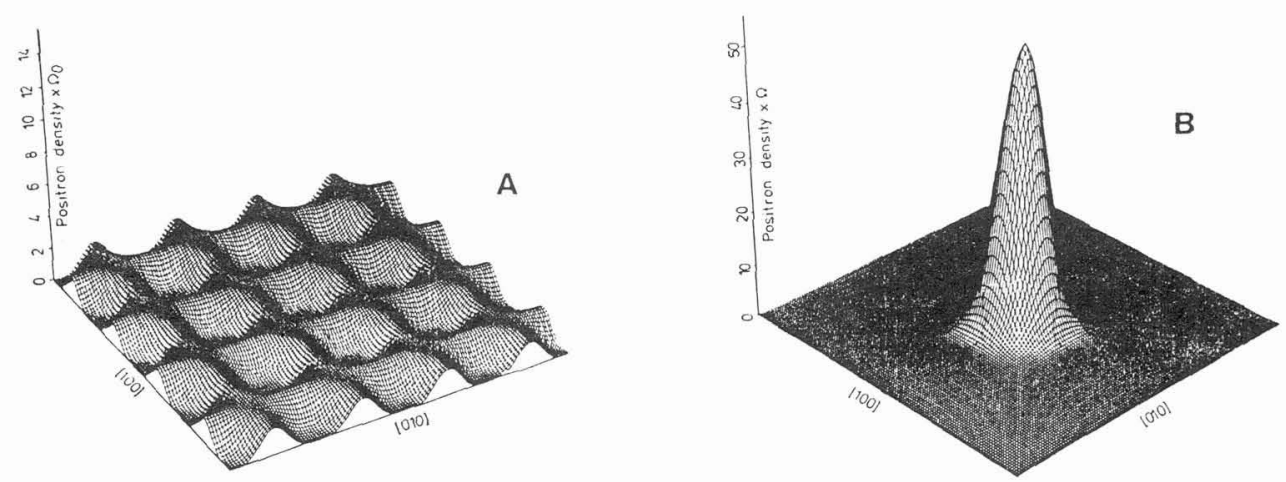

Fig.2. Calculated positron densities in tungsten. A) The positron in the perfect lattice in a Bloch function state. The density vanishes at the cores of the positive ions and has maxima in the interstices. B) The positron trapped in a vacancy where it is strongly localized. From [18].

The annihilation characteristics depend on the type of defect in which the positron is trapped. Hence it is possible to a large extent to differentiate between different types of defects, and from the rates with which the defects trap positrons, defect concentrations can be derived. For example, for positrons trapped in vacancy clusters their lifetime will generally increase with cluster size as illustrated by the calculated curves shown in Fig. 3 [19]. The figure also shows the strong influence of impurities (in this case helium) on the lifetime of the trapped positrons. In fact, theoretical calculations can reliably predict lifetimes for positrons trapped at defect clusters of varying size, configuration and impurity content [19-21]. This will be further illustrated in Sec. 4. 


\subsection{Positrons in semiconductors and ceramics}

Positron lifetimes in perfect bulk crystals of semiconductors and ceramics are in the same range as found for metals [22-25]. However, the defect population in these materials is more complex than in metals, mainly because defects may exist in different charge states and since they may be associated with different, non-equivalent, lattice sites (in the compound materials: ceramics and non-elemental semiconductors). Consequently positron-defect interactions are more complicated and less well studied than in metals, although in the most recent years PAS studies of defects in semiconductors have advanced the understanding very rapidly as evidenced by other contributions at this workshop. More systematic PAS investigations of defects in ceramics have also begun recently (see Section 4) and are likely to advance in a similar successful way as the semiconductor work [24-26]. Since semiconductors are the subject of several contributions at this workshop, we shall not discuss this topic further here.

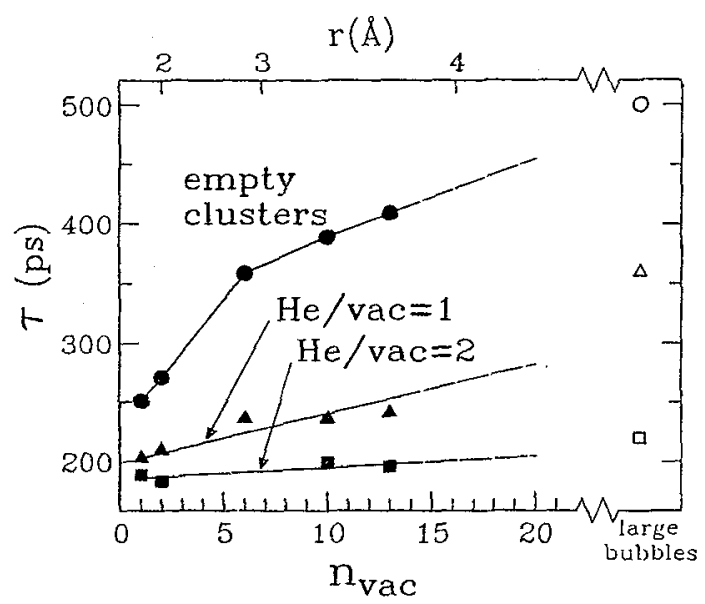

Fig.3. Calculated positron lifetimes in empty and Hefilled 3-dimensional vacancy clusters (bubbles) in aluminium as functions of the number of vacancies in the cluster. "He/vac $=1$ (or 2 )" means that the number of $\mathrm{He}$ atoms in the cluster equals the number of vacancies (or is twice the number of vacancies). From [19].

\subsection{Positronium in organic solids}

In most organic solids a fraction of the injected positrons will form a bound state with an electron, the so-called positronium atom (Ps), a light analog to the hydrogen atom, before annihilation. Two ground states of Ps exisist. The singlet or para-Ps state, in which the positron and electron spins are antiparallel, has an intrinsic lifetime against 2 $\gamma$-decay of 125 psec. The triplet or ortho-Ps state has parallel particle spins and therefore, in vacuum, it can only decay by $3 \gamma$ annihilation with the much longer mean lifetime of 142 nsec [27-29]. Thus, in materials where Ps is formed, three different "positron particles" exist, viz. the "bare" positron, the para-Ps, and the ortho-PS, each of which may be found in more than one state (e.g. "free", or bound to molecules or defects).

The characteristics of Ps in a condensed material are different from those for Ps in vacuum because of the overlap of the surrounding electrons with the Ps atom [28]. Two effects of this overlap are particularly important. One effect is the so-called pick-off process by which the positron in ortho-Ps annihilates with one of the surrounding

electrons which has its spin opposite to that of the positron. In this process two $\gamma$-quanta are emitted and the ortho-Ps lifetime is strongly reduced from the 142 nsec vacuum value to a few nsec or less. The exact magnitude of the lifetime is determined by the amount of overlap between the positron in ortho-Ps and the surrounding electrons (similar to Eq. (2)). The ortho-Ps lifetime is thus a measure of the electron density sampled by the Ps atom.

The second important effect of the Ps overlap with the surrounding electrons in condensed material is a repulsive (exchange) interaction between these electrons and the electron in Ps. Because of this repulsion, Ps atoms will tend to occupy regions of less than average electron density in a material. Such regions may for example be vacancies or clusters of vacancies in crystals, or free volume in polymers. For ortho-Ps, trapping in such regions will lead to a longer 
lifetime because of a reduction in the pick-off rate resulting from the lower electron density.

\section{EXPERIMENTAL TECHNIQUES}

Generally speaking three experimental techniques have been dominating in PAS studies of bulk materials, viz. the lifetime, the angular correlation, and the Doppler broadening techniques. We shall briefly describe these three techniques below. They are all based on nuclear spectroscopy by which the emitted $\gamma$-quanta are detected. More detailed discussions of the techniques may be found in Refs. [16, 30, 31].

For all three techniques the sources of the positrons are in most cases radioactive isotopes such as ${ }^{58} \mathrm{Co},{ }^{64} \mathrm{Cu},{ }^{68} \mathrm{Ge}$ and ${ }^{22} \mathrm{Na}$ which emit positrons $\left(\mathrm{e}^{+}\right.$or $\left.\mathrm{\beta}^{+}\right)$. For lifetime measurements ${ }^{22} \mathrm{Na}$ is particularly useful, because a $1.28 \mathrm{MeV} \gamma$-quantum is emitted essentially simultaneously with the positron, thus signalling the "birth" of the positron. This makes it possible to measure the positron lifetime as we shall now see.

\subsection{Positron Lifetime Technique}

The principle of a positron lifetime spectrometer is shown in Fig. 4. The source is often made by drying a droplet of a ${ }^{22} \mathrm{NaCl}$ aqueous solution on a thin foil $\left(\sim 1 \mathrm{mg} / \mathrm{cm}^{2}\right)$ or directly on the sample. Typical source strengths are $1-50 \mu \mathrm{Ci}$. The source is then sandwiched between two pieces of the sample which are thick enough to ensure that all positrons are stopped in the sample. One of the two detectors (including the connected electronics) detects the $1.28 \mathrm{MeV} \gamma-$ quantum, i.e. signals the emission of the positron, while the other detector detects one of the $511 \mathrm{keV}$ annihilation $\gamma$-quanta, i.e. signals the disappearance of the positron. The time difference between the two detectors signals, i.e. the lifetime of the positron, is measured by the Time-to-Amplitude Converter, and this information is stored in the multichannel analyser (MCA). Typically $10^{6}-10^{7}$ annihilating positrons are recorded and the MCA produces a histogram showing the distribution of positron lifetimes, normally referred to as the lifetime spectrum. Fig. 5 shows examples of such spectra (see also Section 5).

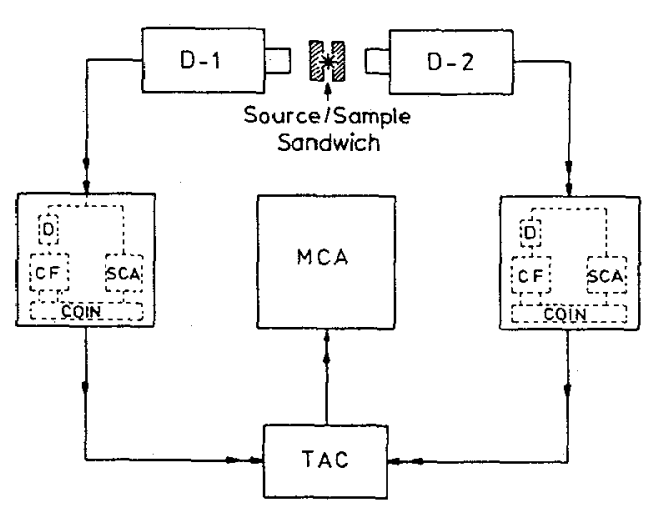

Fig.4. Schematic diagram of a simple, modern lifetime spectrometer. The source is sandwiched between two samples. D-1 and D-2 are two detectors. DISCR are simultaneous time and energy discriminators as indicated inside the boxes, TAC is a time-toamplitude converter, and MCA a multi-channel analyzer.

If the positrons all annihilate from the same state (e.g. bulk Mo) the lifetime spectrum consist of only one decaying exponential (although somewhat broadened by the finite resolution of the spectrometer, see Fig. 5). However, positrons may after slowing down exist in different states (e.g. free $\mathrm{e}^{+}$and Ps), or a transition from one state to another (e.g. trapping into defects) may take place. In such cases positrons will annihilate with different rates determined by their surroundings, and a lifetime spectrum will contain more than one component:

$$
N(t)=\left[\Sigma_{i} A_{i} \exp \left(-\lambda_{i} t\right)\right] * P(t)
$$

Where $*$ symbolizes convolution of the ideal spectrum with the spectrometer time resolution function, $\mathrm{P}(\mathrm{t})$. The two longlived spectra in Fig. 5 consist of more than one component. In a 
lifetime spectrum the physics information is contained in the values of $\lambda_{i}$ and $A_{i}$ ( the latter more often represented by the intensity, $I_{i}=\left(A_{i} / \lambda_{i}\right) / \Sigma\left(A_{i} / \lambda_{j}\right)$, the normalized area under the $i^{\prime}$ th component). By fitting the model function, Eq. (3), to a measured spectrum the values of $I_{i}$ and $\lambda_{i}$ may be extracted [32]. Recently, new types of codes are being developed that can analyze a spectrum consisting of a distribution of lifetimes rather than a set of discrete components (as in Eq.(3)) $[33,34]$.

\subsection{Angular Correlation Measurements}

Figure 6 shows the principle of a conventional angular correlation apparatus. It is used to measure the angular (and hence momentum) distribution of the two $511 \mathrm{keV}$ annihilation quanta (see Section 2). The source is typically of a strenght of 1-50 $\mathrm{mCi}$. It shines positrons down on the sample. The directions of the two emitted photons are defined by collimators which are narrow slits in lead blocks. Typically the sample to detector distance is a couple of meters, and practical angular resolutions will be $0.5-1 \mathrm{mrad}$. The slits closest to the detectors are long slits perpendicular to the plane of the drawing, and the detectors long scintillation crystals mounted just behind the slits, each of them coupled to one or more photomultiplier tubes. One of the detectors (including the collimator) is fixed, while the other one can move to cover angles $\Theta$ in the range of about -30 $\mathrm{mrad}$ to $30 \mathrm{mrad}$. The following electronics count the number of pairs of $511 \mathrm{keV}$ annihilation quanta in a given time as a function of the angle $\Theta$. The data are stored

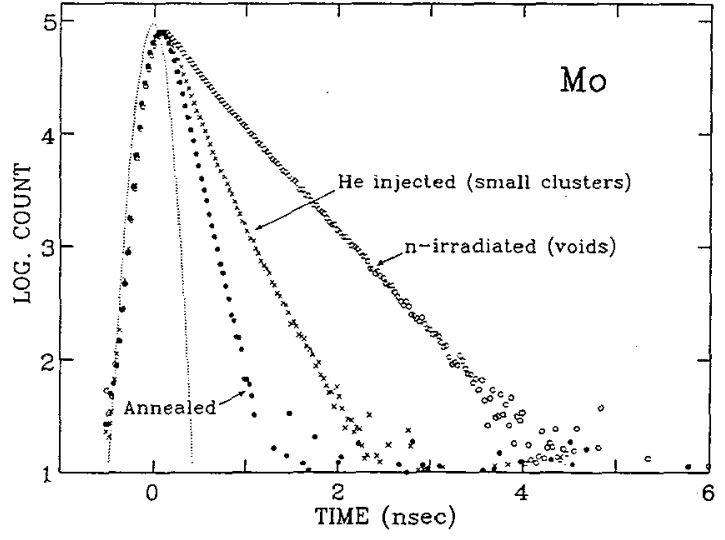

Fig.5. Positron lifetime spectra for three molybdenum samples after subtraction of a constant background. The slopes on the right-hand side of the spectra equal the positron annihilation rates (inverse lifetime). A clear difference is seen between the lifetimes of positrons annihilating in bulk Mo ("Annealed"), trapped in small He-filled vacancy clusters ("He injected"), and trapped in large voids ("n-irradiated"). The dotted curve is the time resultion curve of the lifetime spectrometer.

in the data collection unit which is often a computer, that also controls the measurement. The resulting distribution of angles between the annihilation quanta is called an angular correlation or ACAR (Angular Correlation of Annihilation Radiation) curve. Typical measuring times for a whole curve are from a few hours to a day. Such curves are shown in Fig. 7.

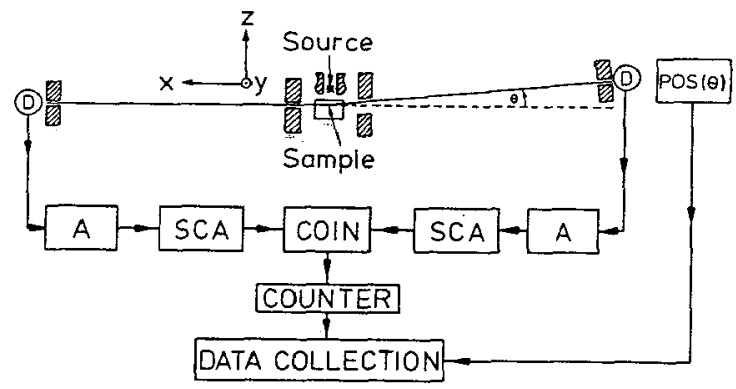

Fig.6. Schematic diagram of a (1-Dimensional) angular correlation apparatus. The source is external to the sample. D represents long scintillation detectors perpendicular to the drawing with lead collimators in front of them. The DATA COLLECTION unit. will often be a computer. POS $(\Theta)$ measures the angle $\Theta$. 
The ACAR apparatus shown in Fig. 6 is a so-called 1-dimensional machine, since it only measures one component of the momentum, viz. the $z$-component, $p_{z}$, but integrates over $p_{x}$ and p $\mathrm{y}^{\prime}$ i.e. $N_{1 D}\left(p_{z}\right)=\iint N_{3 D}\left(\vec{p}_{T}\right) d p_{x} d p_{y}$. Here $N_{3 D}$ is the 3-dimensional momentum distribution. In several laboratories however, so-called 2-dimensional ACAR machines have been developed $[35,36]$. In these, $\gamma$-detectors which are position sensitive in two dimensions are used.Thus, the above integration is only over $\mathrm{p}_{\mathrm{x}}$ and a 2-dimensional momentum distribution is obtained, viz.: $N_{2 D}\left(p_{y^{\prime}} p_{z}\right)=\int N_{3 D}\left(\bar{p}_{T}\right) d p_{x}$. Such, much more detailed, momentum measurements have only been used to a limited extent in defect studies (the contribution by Manuel at this workshop [36] representing one exception).

ACAR curves contain information about the electronic structure in the surroundings of the positron. This is more detailed information than contained in lifetime spectra in which each positron state is characterized by only one number (the lifetime, $\tau$ ). On the other hand it may be difficult to extract the additional information, since in most cases the functional forms of the curves are not known (as they are for lifetime spectra). This is particularly true when positrons annihilate from several different states, thus giving rise to a composite ACAR curve. Ways of analysis are discussed in e.g. [32,36]. A simple way of data treatment is to calculate one or more "shape parameters", e.g. the normalized height of the curve for $\Theta=0$. This does not make use of the whole curve, but may be sufficient in many applications, e.g. in defect studies. Similar ways of analysis are often used for Doppler broadening spectra and will be further illustrated below.

\subsection{Doppler Broadening Measurements}

If the momentum of the annihilating pair has a component, $p_{x}$, in the direction of the emitted $\gamma$ quanta, the frequencies of these quanta and hence their energies will be Doppler shifted [6]. The energy shift is:

$$
\Delta \mathrm{E}=c p_{\mathrm{x}} / 2,
$$

one quantum having the energy $E_{o}+\Delta E$, the other one $E_{o}-\Delta E$, where $E_{o}=m_{0} c^{2}=511 \mathrm{keV}$. Equation (4) shows that $\Delta E$ is proportional to $p_{x}$. Hence, by measuring the distribution of $\Delta \mathrm{E}$ one

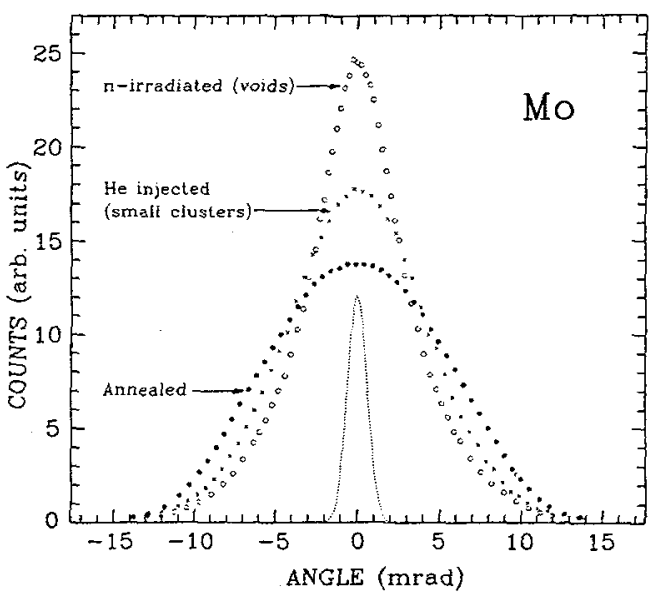

Fig.7. Angular correlation (ACAR) curves for three molybdenum samples (equivalent to those used for Fig. 5). A clear narrowing of the curves is seen when positrons are trapped in small vacancy clusters ("He injected") or in large voids ("n-irradiated"). This is because annihilation in these cases takes place with electrons of lower momentum than it does in bulk Mo ("Annealed"). The curves are normalized to the same area. The dotted curve shows approximately the angular resolution of the apparatus. obtains the distribution of one component of the momentum, just like in 1-dimensional ACAR measurement. Such a measurement can be done with a solid-state detector (intrinsic germanium $(\mathrm{Ge})$ or lithium drifted germanium $(\mathrm{Ge}(\mathrm{Li})$ ) of good resolution (about $1.0 \mathrm{keV}$ FWHM at $511 \mathrm{keV}$ ). A "Doppler broadening spectrometer" is shown schematically in Fig. 8. The signal from the detector is amplified and recorded by the MCA. On expansion of the energy scale in the region around $511 \mathrm{keV}$, spectra like the ones shown in Fig. 9 are obtained. 


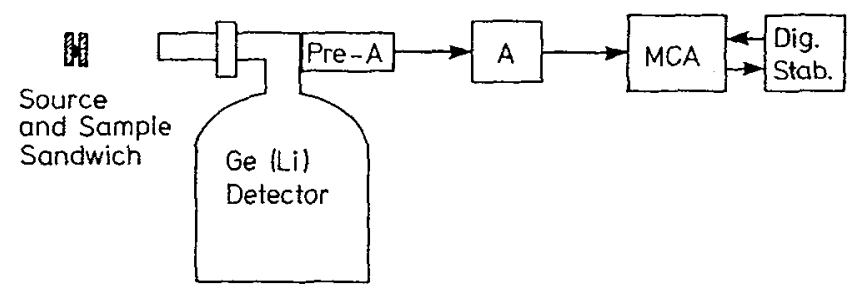

Fig.8. Schematic diagram of a Doppler broadening spectrometer. The source is sandwiched between the two samples. $\mathrm{Ge}(\mathrm{Li})$ represents the solid-state detector cooled by liquid nitrogen. The amplified signal is recorded in a multichannel analyzer that often is connected to a digital stabilizer to secure high stability.

By comparison with Fig. 7 it is seen that the resolution in Fig. 9 is much poorer than in Fig. 7. Since $1 \mathrm{keV}$ is equivalent to $3.914 \mathrm{mrad}$ the resolution curve of a Doppler broadening spectrometer is typically equivalent to $4 \mathrm{mrad}$. This is at least five times broader than the resolution of a typical angular correlation apparatus. Advantages of the Doppler

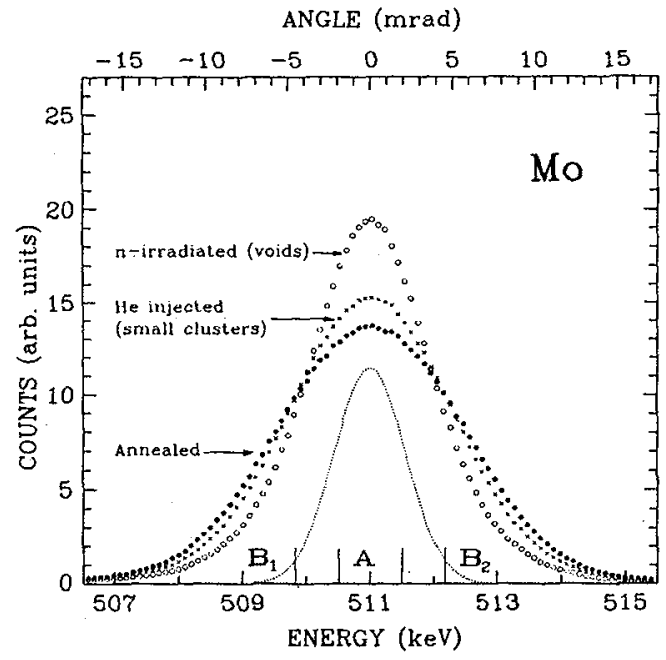

Fig.9. Doppler broadening spectra for three molybdenum samples (equivalent to those used for Figs. 5 and 7). All three curves are normalized to the same area. The dotted curve is the resolution curve of the spectrometer. For comparison with Fig. 7 a $\Theta$-scale is shown at the top which is equivalent to the energy scale at the bottom. The hatched regions show typical integration regions for determination of $S$ and $W$ parameters. compared to the angular correlation technique are that only weak sources (the same as for lifetime measurements) are needed, and that the experimental set-up is simpler, e.g. there are no stringent requirements on the positron of the detector with respect to the source/sample arrangement. Normally a higher countrate is easily obtained so that a spectrum can be measured in a couple of hours or less.

To obtain information from Doppler broadening spectra, in most cases a simple analysis is preferred by which a spectrum is characterized by only one or two parameters. These are often called $S$ and $W . S$ is the area under the spectrum in a narrow energy range symmetrical around $511 \mathrm{keV}$ (A, hatched in Fig. 9), while $W$ is the sum of the areas under the "wings" of the spectrum, i.e. in two regions symmetrical around $511 \mathrm{keV}\left(\mathrm{B}_{1}+\mathrm{B}_{2}\right.$, hatched in Fig. 9), both $S$ and $W$ being normalized to the total area of the spectrum. Instead of $S$, sometimes $\mathrm{H}$ is used which is defined as the normalized peak height of the spectrum. Thus, the narrower the spectrum is, the larger is $S$ and the smaller is $W$. When looking at variations of $S$ and $W$ caused by positron trapping in defects, it will often be useful to consider also a defect specific parameter, viz. $R=\Delta S / \Delta W$ (where $\Delta S$ and $\Delta W$ are the changes in $S$ and $W$ compared to a reference 
spectrum) [38] or - equivalently - a plot of $\Delta S$ vs. $\Delta W$ [39]. More detailed discussions of the Doppler broadening technique can be found in e.g. [16,31].

\section{DEFECTS IN MATERIALS}

As discussed in Section 2 positrons may become trapped in defects in materials, notably vacancies and clusters of these and other regions of less than average density. In the following a few selected examples of this will be shown. First, however, a simple model, the so-called trapping model, will be described. This model is often used to extract quantitative information about defects from PAS data [6,7].

\subsection{Trapping model}

In its simplest version the model assumes that positrons (or Ps atoms) initially are in the bulk of the material where they annihilate with the rate $\lambda_{b}=\tau_{b}{ }^{-1}$. From the bulk they may become trapped in one type of defects with a trapping rate $\kappa$. For a trapped positron the annihilation rate is $\lambda_{\mathrm{d}}=\tau_{\mathrm{d}}{ }^{-1}$. With these assumptions we have the following rate equations for the disappearance of positrons in the bulk and in the trap:

$$
\begin{aligned}
& \frac{d P_{b}(t)}{d t}=-\lambda_{b} P_{b}-\kappa P_{b} \\
& \frac{d P_{d}(t)}{d t}=-\lambda_{d} P_{d}+\kappa P_{b}
\end{aligned}
$$

where $P_{b}$ and $P_{d}$ are the probabilities that the positron is in the bulk and in the trap, respectively. The solutions of the equations result in a lifetime spectrum having two exponential components with the lifetimes:

$$
\tau_{1}=\left(\lambda_{b}+\kappa\right)^{-1}, \tau_{2}=\lambda_{d}^{-1}
$$

and relative intensities:

$$
I_{1}=1-I_{2}, I_{2}=\kappa /\left(\lambda_{b}-\lambda_{d}+\kappa\right)
$$

The parameters defined for momentum measurements in Section 3, viz. H, S and W (as well as the mean lifetime $\bar{\tau}=I_{1} \tau_{1}+I_{2} \tau_{2}$ ) all vary with $\kappa$ in the same way:

$$
F=\left(\lambda_{b} F_{b}+\kappa F_{d}\right) /\left(\lambda_{b}+\kappa\right)
$$

where $F_{b}$ and $F_{d}$ are the characteristic parameter values in the bulk and in the defect, respectively. For increasing trapping rate, $F$ changes in a sigmoidal way from $F_{b}$ at low $\kappa$ to $F_{d}$ at high $\kappa$ values. For a measured value of $\mathrm{F}$, the trapping rate can be determined from $\mathrm{Eq}$. (7). As discussed in e.g. [6,7], the above equations can be expanded to describe more complicated trapping situations which occur, if e.g. more than one type of positron trap exists and/or detrapping can take place. Normally it is a good assumption that the trapping rate is proportional to the defect concentration, $C$, i.e.

$$
\kappa=\mu C
$$

where $\mu$ is called the specific trapping rate for the particular type of defect. For vacancies $\mu$ is of the order of $10^{15} \mathrm{sec}^{-1}$ [17], so changes in lifetimes and intensities (Eqs. $(5,6)$ ) can be detected for relative vacancy concentrations as low as $10^{-7}-10^{-6}$. For three-dimensional vacancy clusters, the specific trapping rate increases with cavity size as illustrated for aluminium in Fig. 10. 


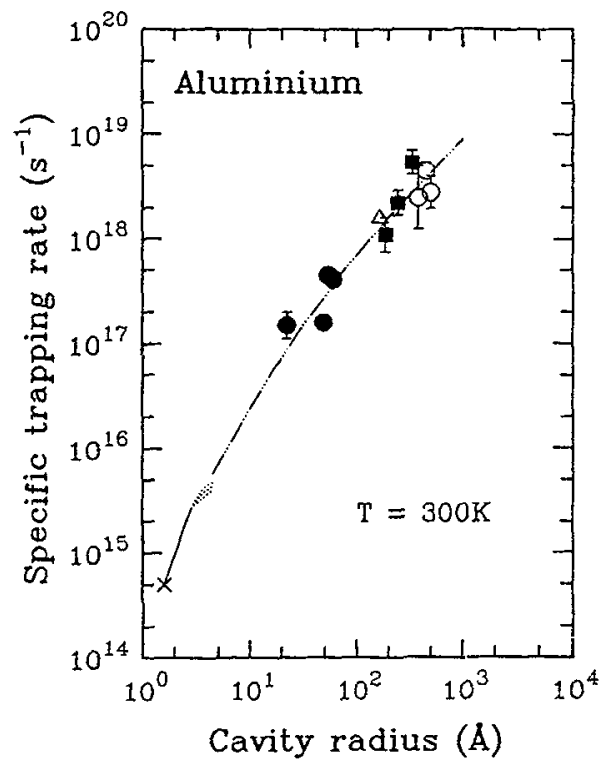

Fig.10. The variation of the specific positron trapping rate, $\mu$, with cavity radius for 3 dimensional vacancy clusters. From [40].

\subsection{Equilibrium vacancies in metals}

A number of PAS studies have been carried out to determine the vacancy formation enthalpies for various metals, not least for metals with high melting points which are difficult to study with other methods, see e.g. [41-44]. This is illustrated in Fig. 11 which shows the variation of a Doppler broadening parameter with temperature. At low temperatures a slow linear change takes place (due to thermal expansion). Above about $0.6 \mathrm{~T}_{\mathrm{m}}$ the vacancy concentration reaches $10^{-7}-10^{-6}$ and trapping of positrons in vacancies begins, leading to steep changes of the plotted parameters. At the highest temperatures, close to the melting points, the vacancy concentrations are high enough $\left(10^{-4}\right.$ $\left.10^{-3}\right)$ to trap almost all positrons, and saturation takes place. By fitting the curves in Fig. 11 to the "trapping model" (Eq. (7)), the variation of the trapping rate $\kappa$ and hence the vacancy concentration C (Eq. (8)) can be obtained. From this the vacancy formation enthalpy can be derived $[42,43,45]$.

Vacancy formation enthalpies have also been determined for a number of alloys and for intermetallic compounds, see e.g. [46,47].

\subsection{Non-equilibrium vacancies in metals. Clustering and interaction with impurities}

A number of studies have been caried out by PAS of the formation (by deformation, irradiation or quenching at low temperatures) and subsequent annealing behaviour of dislocations, vacancies and vacancy clusters (including voids) and the influence of impurities on this behaviour, see e.g. $[1-5,38,40,48-59]$.

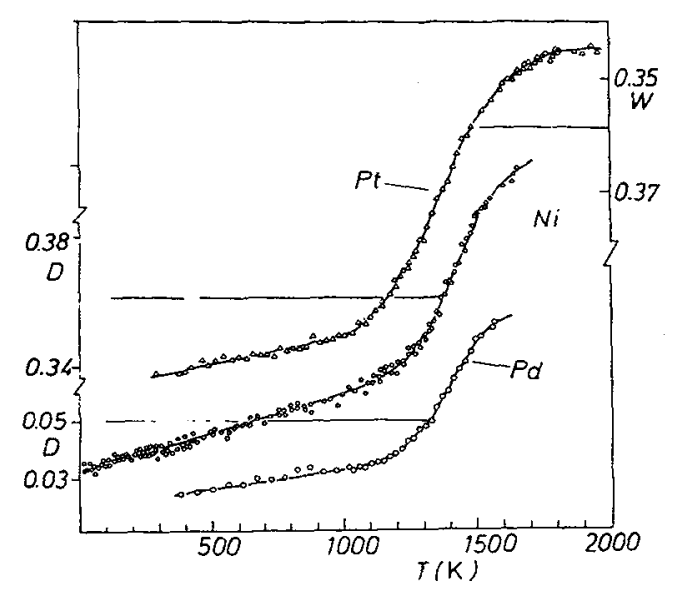

Fig.11. The temperature dependence of the Doppler broadening parameters $W$ for $\mathrm{Pt}$ and $\mathrm{D}(=\mathrm{S}-\mathrm{W})$ for $\mathrm{Ni}$ and $\mathrm{Pd}$. From [45].
Let us illustrate this with one example, viz. niobium, both pure and containing high concentrations (per cent) of hydrogen, that were electron irradiated at low temperatures $(21 \mathrm{~K})$ to create Frenkel pairs. The annealing behaviour is shown in Fig. 12 [54]. At low temperatures, in the as-irradiated state, only one component, due to vacancies, was found. In pure $\mathrm{Nb}$ (Nb1)the lifetime was $210 \mathrm{ps,} \mathrm{while} \mathrm{in} \mathrm{hydrogen}$ containing $\mathrm{Nb}(\mathrm{Nb} 2)$, the lifetime was only 170 ps. Theoretical calculations strongly suggest that this reduction in the lifetime is due to decoration of the vacancies by 1-2 hydrogen atoms [54]. On annealing to $220 \mathrm{~K}(\mathrm{Nb} 1)$ and $380 \mathrm{~K}$ (Nb2) a long-lived component $\tau_{2}, \mathrm{I}_{2}$ appeared (which also resulted in a strong increase of $\bar{\tau}$ ). This gives clear evidence of vacancy clustering. Thus, in pure $\mathrm{Nb}$, vacancy migration (in the so-called stage III) takes place at about $220 \mathrm{~K}$, but it can be shifted to higher temperatures if the vacancies become decorated 
with hydrogen. Another interesting observation is that the vacancy clusters in $\mathrm{Nb} 2$ are rather stable up to at least $700 \mathrm{~K}$ (since $\mathrm{I}_{2}$ decreases only slowly) which is not the case in the pure $\mathrm{Nb}$. Thus, apparently hydrogen plays a role in stabilizing the vacancy clusters [54]. The nature and temperature of stage III has been the subject of much discussion in the literature. Part of the problem has been the potential strong influence of impurities on the vacancy migration temperature. In a number of cases, PAS measurements have clarified this influence, e.g. $[51,53,54]$.

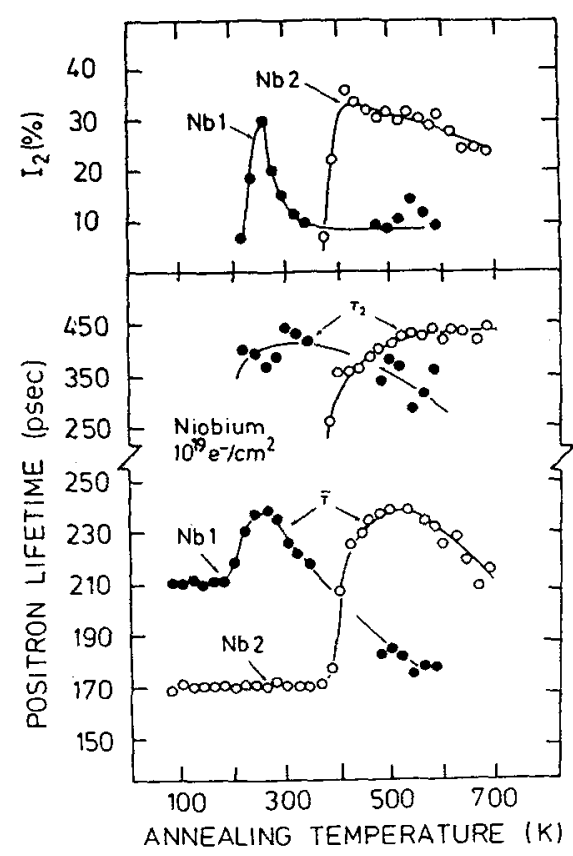

Fig.12. Annealing of electron irradiated pure $(\bullet)$ and hydrogen containing $(0) \mathrm{Nb}$. The average positron lifetime $\bar{\tau}$ (Sec.4.1.) and the long-lived component $\tau_{2}, I_{2}$ are shown as functions of annealing temperature. The appearance of the long-lived component at $220 \mathrm{~K}(\mathrm{Nb} 1)$ is due to vacancy migration and clustering in pure $\mathrm{Nb}$. For $\mathrm{Nb} 2$ the clustering is shifted to $380 \mathrm{~K}$ due to hydrogen impurities. From [54].

\subsection{Gas bubbles in metals}

Unless special care is taken to avoid it, most metals will contain a certain concentration of gas. Even small gas amounts may in some metals influence their properties (as illustrated in Fig. 12). For this reason and in order to gain a fundamental understanding of gas behaviour, a number of applied and fundamental studies of gases in metals have been carried out [60-62].

The special class of inert gases do not react chemically with metals; in fact, there is a strong repulsion between an inert gas atom and a metallic matrix, and hence there is a very strong tendency for rare gases in metals (arising from implantation or nuclear reactions during irradiation) to form complexes with vacancies or vacancy clusters. Complexes that contain many vacancies and gas atoms are called gas bubbles. The creation and behaviour of such bubbles have been looked at in many PAS-studies, see e.g. [19,40,55-59]. As an example of this, let us show (in Fig. 13) some lifetime results for $\mathrm{Cu}$ containing a high concentration ( 3 at \%) of krypton [56].

Below $300^{\circ} \mathrm{C}, \tau_{3}$, that arises from positrons trapped in $\mathrm{Kr}$-bubbles, is constant, equal to about 
250 ps. A relation between positron lifetime and the average $\mathrm{Kr}$-density in the bubbles, $\mathrm{n}$, has been established theoretically (viz.: $\tau_{3}=500-92 \times n$, where the units for $\tau_{3}$ are psec and for $n$ $\left.10^{28} \mathrm{~m}^{-3}\right)[19]$. Using this, a lifetime of $250 \mathrm{ps}$ is equivalent to $\mathrm{Kr}$ density of $2.7 \times 10^{28} \mathrm{~m}^{-3}$. On annealing above $300^{\circ} \mathrm{C}, \tau_{3}$ increases to reach $\sim 400$ ps at $800^{\circ} \mathrm{C}$, equivalent to a $\mathrm{Kr}$ density of $1.1 \times 10^{28} \mathrm{~m}^{-3}$. This reduction in density comes about because the bubbles start to migrate, coalesce

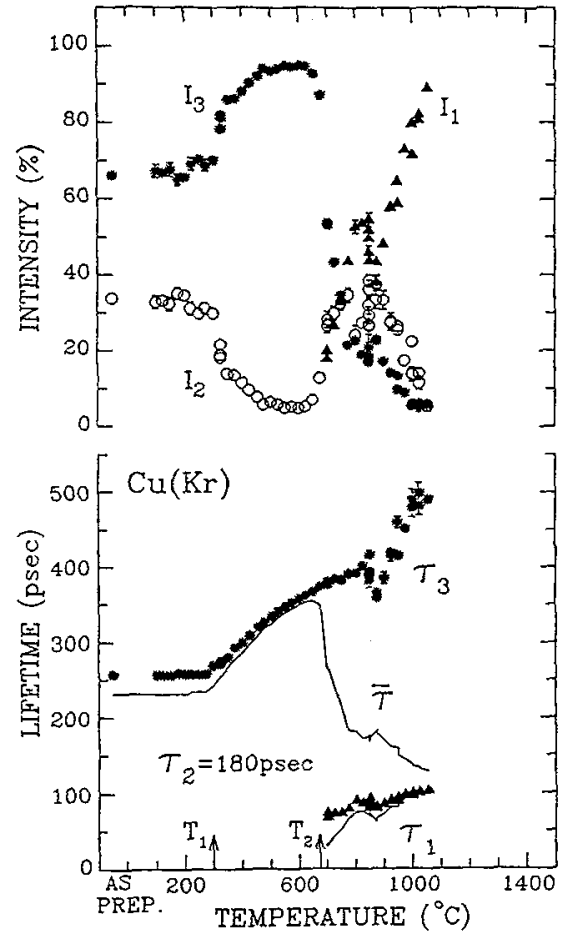

Fig.13 Positron lifetime parameters as functions of annealing temperature for Cu containing $\sim 3$ at $\% \mathrm{Kr}$. The longlived components are associated with $\mathrm{Kr}$-bubbles $\left(\tau_{3}, \mathrm{I}_{3}\right)$, and with small vacancy clusters containing $\mathrm{KI}_{\mathrm{r}}\left(\tau_{2}, \mathrm{I}_{2}\right)$. The lifetime $\tau_{3}$ is a measure of the $\mathrm{Kr}$ density in the bubbles. From [56].

and grow in sizedue to an influx of thermal vacancies. The migration is probably initiated by small vacancy- $\mathrm{Kr}$ clusters that become mobile at about $300^{\circ} \mathrm{C}$ (evidenced by the reduction in $\left.I_{2}\right)[56,63]$. At about $700^{\circ} \mathrm{C}$ a strong decrease of the intensity $I_{3}$, (and therefore also the mean lifetime $\bar{\tau}$ ) takes place because a large fraction of the bubbles coalesce and form open channels to the surface. Most of the $\mathrm{Kr}$ escapes through the channels and the bubble density drops drastically [56].

The ability of PAS to provide information both about gas density and about defect concentration makes it possible to derive also bubble sizes, as discussed in [55,57-59]. In studies like those, the clear advantages of PAS compared to electron microscopy are that PAS is non-destructive, it gives information about average gas densities in bubbles, and submicroscopic cavities can be detected. A disadvantage is that PAS is an averaging technique, i.e. it does not produce for example detailed size distributions of cavities and it may in some cases be difficult to separate components arising from different types of defects.

\subsection{Ceramics}

A number of PAS Studies have been carried out on ceramics, partly inspired by the discovery of the new ceramic superconductors. Many of the investigations have been concentrated on 
defects in the ceramics, see e.g. [4,5,23-26,64-65]. Generally speaking, positrons will not, or only weakly, be bound at anion vacancies because of their positive charge, while the binding at cation vacancies normally will be much stronger [64]. Therefore, trapping of positrons will mainly be observed at negative cation vacancies, or complexes of cation and anion vacancies, although clusters of anions may apparently also provide traps for positrons [65]. Furthermore, each defect may exist in different charge states which may have different specific trapping rates and give rise to different positron lifetimes (and momentum distributions). This is illustrated by the lifetime results shown in Fig. 14 [25].

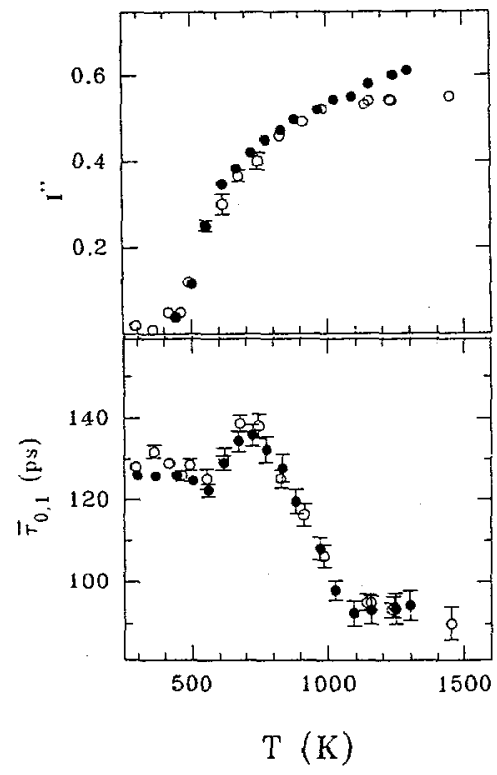

Fig.14 The variation of positron lifetime parameters with temperature for $\mathrm{NiO}$ at two different oxygen partial pressures (o: $1 \mathrm{~Pa}, \bullet 10^{5} \mathrm{~Pa}$ ). $\mathrm{I}^{\prime \prime}$ is the intensity of a 205ps component, while $\bar{\tau}_{0,1}$ is the mean of two shorter lifetimes. From [25].
A long lifetime component (205ps, intensity $\left.I^{\prime \prime}\right)$ is associated with positrons trapped at doubly charged Ni-vacancies, while the short-lived component $\bar{\tau}_{0,1}$ is an unresolved mean lifetime of positrons in the bulk crystal and trapped in singly charged Nivacancies [25]. At low temperatures $(<700 \mathrm{~K})$ the increase I" and the variation of $\bar{\tau}_{0,1}$ are associated with a thermally activated change of prexisting $\mathrm{Ni}$ vacancies from neutral to singly charged and from singly to doubly charged Ni-vacancies. Above $700 \mathrm{~K}$ the further increase in I" and decrease of $\bar{\tau}_{0,1}$ are ascribed to further transformation from singly to doubly charged Ni-vacancies [25]. Only at the highest temperatures can thermal equilibrium between the $\mathrm{NiO}$ crystal and the oxygen atmosphere be established within the measuring time and a decrease of the Ni-vacancy concentration is observed (by the decrease of $I^{\prime \prime}$ ) for low oxygen pressure compared to high pressure [25].

\subsection{Organic solids}

In this section we shall first briefly mention some results obtained for defects in molecular crystals (i.e. crystals where lattice positions are occupied by molecules) and secondly illustrate the application of PAS to obtain information about free volumes in polymers.

In most molecular solids a fraction of the injected positrons forms Ps, as discussed in Section 2. Because Ps is attracted to regions of lower-than-average density it may become trapped in vacancy-type defects in molecular crystals. The trapping leads to an increase of the ortho-Ps lifetime and will normally also be reflected in the measured momentum distribution. A number of molecular crystals (both brittle, plastic and liquid crystals) have been investigated by PAS, see e.g. [66-71]. In a group of these (i.e. ice and the plastic crystals) trapping of Ps into vacancies has been found. A sigmoidal increase of the ortho-Ps lifetime with temperature, similar to the ones shown for metals in Fig.11, has been observed for several crystals and from these data vacancy formation energies have been derived [71]. Studies of ice crystals in which defects have been introduced by doping or irradiation have shown that in some molecular crystals it is also possible to follow the migration and clustering of non-equilibrium vacancies [66,69,72]. Based on these studies it was possible to establish a useful correlation between cavity size in a molecular substance and the lifetime of ortho-Ps trapped in the cavity (for approximately spherical cavities). This is shown in Fig. 15 (see also $[67,70]$ ). Thus, by using this relationship one can in principle 


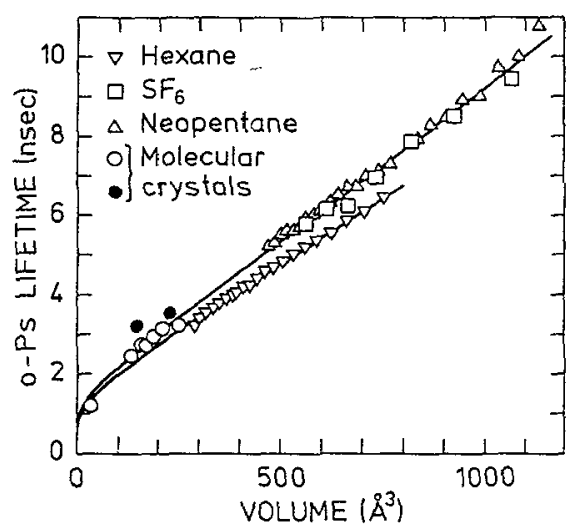

Fig. 15. The relationship between the lifetime of ortho-Ps in an approximately spherical cavity in a molecular substance and the cavity volume. From [66].

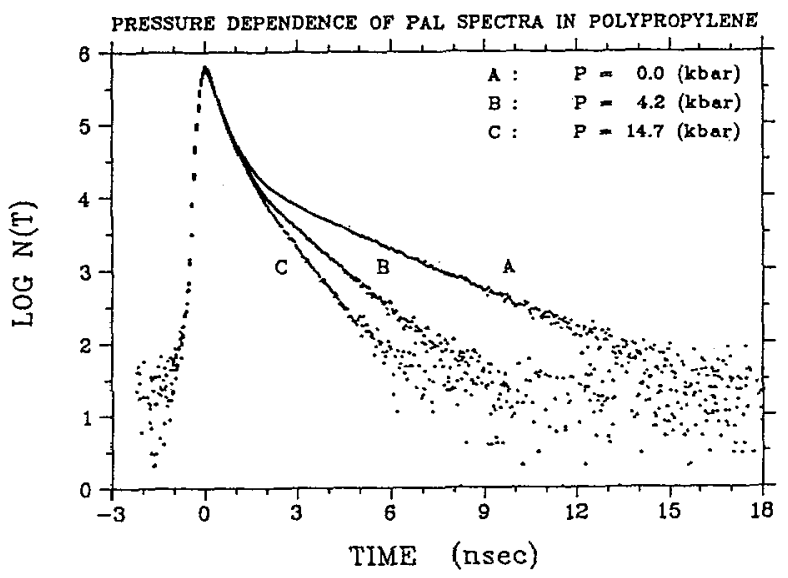

Fig. 16. Positron lifetime spectra for polypropylene under different pressures. The mean orthoPs lifetime decreases from about $2,3 \mathrm{~ns}$ at 0 kbar to about $0,7 \mathrm{~ns}$ at 14,7 kbar. From[73]. obtain information about cavity volumes in molecular substances by measuring ortho-Ps lifetimes (see below). It should also be added that phase transitions (solid-solid and solid-liquid) have been investigated for many plastic and liquid crystals $[67,68]$. Furthermore it should be mentioned that in a number of molecular crystals, especially ordinary brittle crystals, PS seems to be trapped at other defects than vacancies. Further studies are required to determine the exact nature of these defects [67].

For polymers an important quantity that influences the physical properties of the polymer is the so-called free volume which is a measure of the volume not occupied by the molecules. In principle it is possible to obtain information on the sizes of the cavities, that constitute the free volume, by PAS lifetime measurements, since the lifetime of ortho-Ps gives a measure of the volume of the hole in which it is trapped (Fig. 15). Recently, a number of studies along these lines have been carried out, see e.g. [73-76], and the technique is being developed to provide size distributions for the cavities in which ortho-Ps is trapped [33,73-75]. Fig. 16 shows the strong sensitivity of the long ortho-Ps lifetime to pressure induced changes of free volume in polypropylene. Although qualitatively the derived variations of free volume sizes with e.g. temperature or pressure (Fig. 16) clearly represent the correct trends, maybe some caution is needed concerning the exact shapes (and mean values) of the obtained size distributions, as discussed in [75]. This caution 
arises from the uncertainties concerning the dependence of the Ps trapping probability on cavity sizes, the influence of cavity shape, possible chemical reactions of Ps with the molecules, and the data analysis. At present, work is going on to resolve these problems [75].

\section{Conclusion}

In the present paper positron annihilation techniques used for bulk studies were briefly described, both some basic physics principles and the experimental techniques. Emphasis was placed on the sensivity of the techniques to defects in solids. This sensivity was illustrated by a number of examples, for metals, ceramics and molecular solids (semiconductors being dealt with by others at the workshop). For metals in particular it was pointed out that positrons are sensitive to vacancy type defects, the main sensitivity being for sizes from monovacancies up to clusters containing 50-100 vacancies and for relative concentrations of $10^{-4}-10^{-7}$ and below, depending upon the size, thus covering the range from the smallest defects up to sizes visible by electron microscopes. It was exemplified that vacancy - impurity interactions can be observed and that information may be obtained about gas densities in gas bubbles as well as about bubble sizes and densities. Similarly, the possibility to follow changes in the defect population in ceramic materials and to obtain information about free volume in molecular materials was discussed.

Hopefully, the present paper has clearly illustrated why the positron annihilation technique has now become a well-established, non-destructive technique for studies of defects in bulk materials but at the same time a technique which is still being developed and used in new applications.

\section{References:}

[1] Hasiguti R.R. and Fujiwara K., Eds., "Positron Annihilation" (Proc. 5'th Int. Conf. on Positron Annihilation), (Japan Institute of Metals, Sendai, Japan 1979).

[ 2] Coleman P.G., Sharma S.C. and Diana L.M., Eds., "Positron Annihilation" (Proc. 6'th Int. Conf. on Positron Annihilation), (North-Holland, Amsterdam 1982).

[ 3] Jain P.C., Singru R.M. and Gopinathan K.P., Eds., "Positron Annihilation", (World Scientific, Singapore 1985).

[ 4] Dorikens-Vanpraet L., Dorikens M. and Segers D., Eds., "Positron Annihilation" (Proc. 8'th Int. Conf. on Positron Annihilation), (World Scientific, Singapore 1989).

[ 5] Kajcsos Zs. and Szeles Cs., Eds., "Positron Annihilation" (Proc. 9'th Int. Conf. on Positron Annihilation) Materials Science Forum 105-110 (1992).

[ 6] Hautojärvi P., Ed.,"Positrons in Solids" (Topics in Current Physics), (Springer, Berlin 1979).

[7] Brandt W. and Dupasquier A., Eds., "Positron Solid-State Physics", (North-Holland, Amsterdam 1983).

[ 8] Dupasquier A. and Mills A.P., Eds., "Positron Spectroscopy of Solids". (North Holland, Amsterdam 1994), in press.

[ 9] Schultz P.J. and Lynn K.G., Rev. Mod. Phys. 60, (1988) 701-779.

[10] Schultz P.J., Massoumi G.R., and Simpson P.J., Eds., "Positron Beams for Solids and Surfaces". AIP Conf. Proc. 218 (AIP, New York 1990).

[11] Contributions by P. Hautojärvi and by W. Triftshäuser at EIW-12.

[12] Berko S., "Fermi surface studies in disordered alloys: Positron annihilation experiments", in: "Electrons in Disordered Metals and at Metallic Surfaces", P. Phariseau, B. L. Gyorffy, and L. Scheire, Eds. (Plenum, New York 1979) pp. 239-291.

[13] Berko S., "Momentum density and Fermi-surface measurements in metals by positron annihilation, in: "Positron Solid-State Physics", Ref. [7] pp. 64-145.

[14] Mijnarends P.E., "Electron momentum densities in metals and alloys", in: "Positrons in Solids", Ref. [6], pp. 25-88 and "Momentum density in metals and alloys: Theory", in: "Positron Solid-State Physics", Ref. [7] pp. 146-195. 
[15] West R.N., "Positrons as solid state probes", in: "Nuclear Physics Methods in Materials Research", K. Bethge, H. Baumann, H. Jex, and F. Rauch, Eds.(Vieweg, Braunschweig 1980) pp. 234-247, and "Fermi Surface Studies with Positrons" in: "Metallic Alloys: Experimental and Theoretical Perspectives", J.S. Faulkner and R.G. Jordan, Eds. (Kluwer, The Netherlands 1994)

[16] Mackenzie I.K., "Experimental methods of annihilation time and energy spectrometry", in: "Positron Solid-State Physics", Ref. [7], pp. 196-297.

[17] Nieminen R.M. and Manninen M.J., "Positron in imperfect solids: Theory", in: "Positron in Solids", Ref.[6]. pp. 145-195.

[18] Gupta R.P. and Siegel R.W., J. Phys. F. 10 (1980) L7-L13.

[19] Jensen K.O. and Nieminen R.M., Phys. Rev. B 36 (1987) 8219-8232.

[20] Puska M.J. and Nieminen R.M. J. Phys. F. 13 (1983) 333-346.

[21] Hansen H.E., Nieminen R.M., and Puska M.J., J. Phys. F. 14 (1984) 1299-1316.

[22] Corbel C., Mat. Sci. Forum 105-110 (1992) 221-228 and Hautojärvi P. (This workshop).

[23] Puska M.J., Mäkinen S., Manninen M., and Nieminen R.M., Phys. Rev. B 39 (1989) 76667679.

[24] Schaefer H.-E. and Forster M., Mat. Sci. Engineering A109 (1989) 161-167.

[25] Forster M., Claudy W., Hermes H., Koch M., Maier K., Major J., Stoll H. and Schaefer H.E., Mat. Sci. Forum 105-110 (1992) 1005-1012.

[26] Sob M., Contribution at EIW-12.

[27] Berko S. and Pendleton H.N., Ann. Rev. Nucl. Part. Sci. 30 (1980) 543-581.

[28] Dupasquier A., "Positronium like system in solids", in: "Positron Solid-State Physics", Ref. [7] (1983) pp 510-564.

[29] Mogensen O.E., J. Chem. Phys. 60 (1974) 998-1004.

[30] Berko S., "Two-dimensional angular correlation of annihilation radiation experiments", in:"Positron Annihilation", Ref. [1] pp. 65-87.

[31] Smedskjaer L.C. and Fluss M.J., "Experimental methods of positron annihilation for the study of defects in metals", in: "Methods of Experimental Physics", Vol. 21, J.N. Mundy, S.J. Rothmann, M.J. Fluss and L.C. Smedskjaer, Eds. (Academic Press, New York 1983) pp. 77-145.

[32] Kirkegaard P., Eldrup M., Mogensen O.E. and Pedersen N.J., Comput. Phys. Commun. 23 (1981) 307-335, and Kirkegaard P., Pedersen N.J. and Eldrup M., Risø Report M-2740 (1989).

[33] Gregory R.B. and Zhu Y., Nucl. Instr. Meth. A290 (1990) 172-182.

[34] Shukla A., Peter M. and Hoffmann L., Nucl. Instr. Meth. A335 (1993) 310-317.

[35] "Electronic Structure of Matter" in "Positron Annihilation", Ref. [4] pp. 109-136.

[36] Eldrup M., Mackenzie I.K., McKee B.T.A. and Segers D., in "Positron Annihilation", Ref. [4] pp. 216-226.

[37] Fluss M. J., Berko S., Chakraborty B., Hoffmann K.R., Lippel P. and Siegel R.W., J. Phys. F. 14 (1984) pp 2831-2854.

[38] Mantl S. and Triftshäuser W., Phys. Rev. Lett. 34 (1975) 1554-1557.

[39] Liszkay L., Corbel C., Baroux L., Hautojärvi P., Bayhan M., Brinkman A.W. and Tatarenko S., Appl. Phys. Lett. 64 (1994) 1380-1382, and contribution at EIW-12.

[40] Jensen K.O., Eldrup M., Singh B.N., Horsewell A., Victoria M. and Sommer W.F., Mat. Sci. Forum 15-18 (1987) 913-918.

[41] Seeger A., J. Phys F. 3 (1973) 248-294.

[42] Maier K., "Defects in thermal equilibrium: Positron anihilation and other methods", in: "Positron Solid-State Physics", Ref [7], pp. 265-297.

[43] Schaefer H.E., "Thermal equilibrium studies of vacancies in metals by positron annihilation", in: "Positron Annihilation", Ref. [2], pp. 369-380.

[44] Siegel R.W., "Positron annihilation spectroscopy of defects in metals - an assessment", in: "Positron Annihilation", Ref [2], pp. 351-368. 
[45] Maier K. Rein G., Saile B., Valenta P., and Schaefer H.E., in: "Positron Annihilation", Ref. [1] pp. 101-105.

[46] Kluin J.-E. and Hehenkamp Th., Phys. Rev. B 44 (1991) 11597-11608.

[47] Schaefer H.-E., Würschum R. and Bub J., Mat. Sci. Forum 105-110 (1992) 439-450.

[48] Petersen K., "Studies of Nonequilibrium Defects in Metals", in: "Positron Solid-State Physics", Ref. [7] pp. 298-358.

[49] Byrne J.G., Met. Trans. A 10A (1979) 791-807 and Byrne J.G., "Dislocation studies with positrons", in: "Dislocations in Solids", Vol. 6, F.R.N. Nabarro, Ed. (North-Holland, Amsterdam 1983) pp. 265-318.

[50] Eldrup M., Mogensen O.E. and Evans J.H., J. Phys. F 6 (1976) 499-521.

[51] Vehanen A., Hautojärvi P., Johansson J., Yli-Kauppila J. and Moser P., Phys. Rev. B 25 (1982) $762-780$.

[52] Hautojärvi P., Pöllänen L., Vehanen A., and Yli-Kauppila J., J. Nucl. mat. 114 (1983) 250259.

[53] Nielsen B., van Veen A., Caspers L.M., Filius H.A., Hansen H.E. and Petersen K., in: "Positron Annihilation", Ref. [2] pp. 438-440.

[54] Hautojärvi P., Huomo H., Puska M. and Vehanen A., Phys. Rev. B. 32 (1985) 4326-4331.

[55] Jensen K.O., Eldrup M., Singh B.N. and Victoria M., J. Phys. F 18 (1988) 1069-1089.

[56] Jensen K.O., Eldrup M., Pedersen N.J. and Evans J.H., J. Phys F 18 (1988) 1703-1724.

[57] Linderoth S., J. Phys.: Condens. Mat. 1 (1989) SA55-SA66.

[58] Viswanathan B., J. Phys.: Condens. Mat. 1 (1989) SA71-SA76.

[59] Eldrup M., Mat. Sci. Forum 105-110 (1992) 229-248.

[60] Alefeld G. and Völkl J. Eds., "Hydrogen in Metals" (Springer, Berlin 1978).

[61] Ullmaier H., Ed., "Proc. Int. Symp. on Fundamental Aspects of Helium in Metals", Rad. Effects 78 (1983).

[62] Donnell S.E. and Evans J.H., Eds., "Fundamental Aspects of Inert Gases in Solids" (Plenum, New York 1991).

[63] Skov Pedersen J., Eldrup M. and Horsewell A., Submitted to J. Phys.: Cond. Matter

[64] McMullen T., Jena P., Khanna S.N., Li Yi, Jensen K.O., Phys. Rev. B 43 (1991) 10422-10430.

[65] Pareja R, de la Cruz R.M., González R. and Chen Y., Phil. Mag. A 66 (1992) 597-607.

[66] Eldrup M., "On positron studies of molecular crystals" in: "Positron Annihilation", Ref. [2] pp. 753-762.

[67] Goworek T., "Positrons and positronium in molecular solids" in : "Positron Annihilation", Ref. [4] pp. 137-146. and Goworek T., Phys. Stat. sol. (a) 102 (1987) 511-526.

[68] Jain P.C., "Phase transition studies in molecular materials and non-metallic systems" in: "Positron Annihilation", Ref. [4] pp. 153-157.

[69] Eldrup M.:, "Application of the positron annihilation technique in studies of defects in solids" in: "Defects in Solids", A.V. Chadwick and M. Terenzi, Eds. (Plenum, New York 1986) pp. $145-178$.

[70] Schrader D.M. and Jean Y.C., Eds., "Positron and Positronium Chemistry" (Elsevier, Amsterdam 1988).

[71] Lightbody D., Sherwood J.N., and Eldrup M., Mol. Cryst. Liq. Cryst. 96 (1983) 197-210.

[72] Eldrup M., J. Chem. Phys. 64 (1976) 5283-5290. and Eldrup M., Mogensen O.E. and Bilgram J.H., J. Glaciology 21 (1978) 101-113.

[73] Jean Y.C. and Deng Q., J. Polymer Sci. B 30 (1992) 1359-1364.

[74] Jean Y.C., Mat. Sci. Forum 105-110 (1992) 309-316.

[75] Dai G.H., Deng Q., Liu J., Shi H., Huang C.M., and Jean Y.C., J. de Physique IV Colloque 3(4) (1993) 233-239.

[76] Uedono A., Kawano T., Wei L., Tanigawa S., Ban M. and Kyoto M., contribution at EIW12. 